\title{
The Numerical Solution of Heat Problem Using Cubic B-Splines
}

\author{
Duygu Dönmez Demir ${ }^{*}$ Necdet Bildik \\ Department of Mathematics, Faculty of Art and Science, Celal Bayar University, Manisa, 45047, Turkey \\ necdet.bildik@cbu.edu.tr
}

\begin{abstract}
This paper discusses solving one of the important equations in Physics; which is the one-dimensional heat equation. For that purpose, we use cubic B-spline fin ite elements within a Collocation method. The scheme of the method is presented and the stability analys is is investigated by considering Fourier stability method. On the other hand, a comparative study between the numerical and the analytic solution is illustrated by the figure and the tables. The results demonstrate the reliability and the efficiency of the method.
\end{abstract}

Keywords Cubic B-splines Finite Element Method, Collocation Method, Cubic B-splines, Finite Element Method

\section{Introduction}

Consider the one dimensional initial-boundary value problem

$$
\alpha^{2} u_{x x}-u_{t}=0,0 \leq x \leq L, t>0
$$

with initial condition

$$
u(x, 0)=f(x)
$$

and boundary conditions

$$
u(0, t)=u(L, t)=0 \text { for } t>0 .
$$

This problem is one of the well-known second order linear partial differential equation[1-3]. It shows that heat equation describes irreversible process and makes a distance between the previous and next steps. Such equations arise very often in various applications of science and engineering describing the variation of temperature (or heat distribution) in a given region over some time[4]. It can be expressed as the heat flow in the rod with diffusion $\alpha^{2} u_{x x}$ along the rod where the coefficient $\alpha$ is the thermal diffusivity of the rod and $L$ is the length of the rod[5]. In this model, the flow of the heat in one-dimension that is insulated everywhere except at the two end points. Solutions of this equation are functions of the state along the rod and the time $t$. In the past, this problem has been widely worked over a number of years by numerous authors. But it is still an interesting problem since many physical phenomena can be formu lated into PDEs with boundary conditions. The heat equation is of fundamental importance in diverse scientific fields. It is the prototypical

* Corresponding author:

duygu.donmez@cbu.edu.tr (Duygu Dönmez Demir)

Published online at http://journal.sapub.org/xxx

Copyright (C) 2012 Scientific \& Academic Publishing. All Rights Reserved parabolic partial differential equation in mathematics. In probability theory, the heat equation is connected with the study of Brownian motion via the Fokker-Planck equation[6]. Numerical solutions of those equations are very useful to study physical phenomena. One of the linear evolution equation which we deal with the numerical solution is the heat equation[7].

In 1946, Schoenberg first proposed the theory of B-splines[4]. Recurrence relations for the purpose of computing coefficients are given by Cox and de Boor[8,9]. The cubic B-splines collocation method was developed for Burgers' equation and used for the numerical solution of the differential equations in[10,11]. Recently, spline function theory has been extended and developed to solve the differential equations numerically by various papers[12-14]. Furthermore some extraordinary problems has been numerically investigated by finite element methods such as Galerkin method, least square method and collocation method with quadratic, cubic, quintic and septic B-splines [15-17].

Various techniques of both the cubic spline and cubic B-spline collocation methods and their application have been developed to obtain the numerical solution of the differential equations. They possess some of advantages and are worth on using in the numerical techniques. So cubic spline collocation procedures exhibits the following the desirable features: (1) obtained governing system is always diagonal which permits easy algorithms; (2) it provides low computer cost and easy problem formulation. The requirement of the continuity up to the second degree are guaranteed at the mesh points over the domain and the first and second degree of the derivatives are directly evaluated[7,12,18].

In this study the cubic B-splines collocation method is used for solving the heat equation (1) subject to (2) and (3) 
and the solutions are compared with the exact solution[19]. For constructing the cubic B-splines finite element method, we use collocation techniques as it was extensively used in $[7,20,21]$. In the section two, proposed method is presented and it is also given how to apply the collocation method with cubic B-splines finite element technique. In the section three, the stability analysis is investigated considering Fourier stability method. Finally, the numerical results and the related tables are given in the next section.

\section{Collocation Method}

Let us consider the doma in $[0, L]$ that is equally-divided with nodal points $x_{j}$ such that $0=x_{0}<x_{1}<\ldots<x_{N}=L$, i.e., finite elements of length $h=x_{j+1}-x_{j}$ for $j=0, \ldots, N-1$, and also suppose that $\varphi_{m}(x)$ to be cubic B-splines at the nodal points $x_{m}$ for $m=-1, \ldots, N+1$. Using cubic B-splines $\varphi_{m}(x)$, the exact solution $U(x, t)$ is approached by an approximation $U_{N}(x, t)$ such that

$$
U_{N}(x, t)=\sum_{m=-1}^{N+1} \delta_{m}(t) \varphi_{m}(x)
$$

where $\delta_{m}(t)$ is parameter in terms of the time $t$ for $m=-1, \ldots, N+1$ to be identified by the boundary conditions and the collocation conditions.

The cubic B-splines $\varphi_{m}(x)$ are defined in [19].

Considering the approximation function (4) and the cubic B-splines $\varphi_{m}(x)$ defined in (5), the required values of $U_{m}$ and its first and the second derivatives with respect to $x$ at the nodal points $x_{m}$ are identified in terms of $\delta_{m}$ as

$$
\begin{aligned}
& U_{m}=U\left(x_{m}\right)=\delta_{m-1}+4 \delta_{m}+\delta_{m+1} \\
& U_{m}^{\prime}=U^{\prime}\left(x_{m}\right)=\frac{3}{h}\left(\delta_{m+1}-\delta_{m-1}\right) \\
& U_{m}^{\prime \prime}=U^{\prime \prime}\left(x_{m}\right)=\frac{6}{h^{2}}\left(\delta_{m-1}-2 \delta_{m}+\delta_{m+1}\right) .
\end{aligned}
$$

For instance, let us take $\alpha=1, L=1$ and $f(x)=\sin (\pi x)$ in (1) and (2), respectively. There fore the problem (1) subject to (2) and (3) is become

$$
u_{x x}-u_{t}=0,0<x<1, t>0
$$

with initial condition

$$
u(x, 0)=\sin (\pi x)
$$

and boundary conditions

$$
u(0, t)=u(1, t)=0 \text { for } t>0 .
$$

One can obtain the approximate solution for the heat equation namely

$$
U_{x x}-U_{t}=0
$$

by considering the solution of

$$
\left(U_{t}\right)_{m}^{n}+(1-\theta) f_{m}^{n}+\theta f_{m}^{n+1}=0
$$

where

$$
f_{m}^{n}=\alpha^{2}\left(U_{x x}\right)_{m}^{n}
$$

Here $\theta$ is a parameter that when it takes the value 0 , the scheme is so called forward Euler and also if $\theta=1 / 2$, then the scheme is called Crank-Nicholson, and if $\theta=1$, the scheme is so called backward Euler. Then we discretize the time derivative by means of fin ite difference so we have

$$
U_{m}^{n}-U_{m}^{n+1}+\Delta t(1-\theta) \alpha^{2}\left(U_{x x}\right)_{m}^{n}+\Delta t \theta \alpha^{2}\left(U_{x x}\right)_{m}^{n+1}=0
$$


Substituting (6) into (13), we obtain the following difference equation system for the variables $\delta$, which has $n+1$ difference equations with $n+3$ unknown values as

$$
\delta_{m-1}^{n+1}\left(1-\frac{6}{h^{2}} \Delta t \theta \alpha^{2}\right)+\delta_{m}^{n+1}\left(4+\frac{12}{h^{2}} \Delta t \theta \alpha^{2}\right)+\delta_{m+1}^{n+1}\left(1-\frac{6}{h^{2}} \Delta t \theta \alpha^{2}\right)=L_{1}+\Delta t(1-\theta) \alpha^{2} L_{3}
$$

where

$$
L_{1}=\delta_{m-1}^{n}+4 \delta_{m}^{n}+\delta_{m+1}^{n}, L_{2}=\frac{3}{h}\left(\delta_{m+1}^{n}-\delta_{m-1}^{n}\right), L_{3}=\frac{6}{h^{2}}\left(\delta_{m-1}^{n}-2 \delta_{m}^{n}+\delta_{m+1}^{n}\right) .
$$

Then this set of equations is a recurrence relationship of element parameters vector $d^{n}=\left(\delta_{-1}^{n}, \delta_{0}^{n}, \delta_{1}^{n}, \ldots, \delta_{N}^{n}, \delta_{N+1}^{n}\right)$. Using the boundary conditions (9) and eliminating the parameters $\delta_{-1}, \delta_{N+1}$ in (14), then the system may be rewritten as

$$
\begin{aligned}
& U\left(x_{0}\right)=\delta_{-1}^{n+1}+4 \delta_{0}^{n+1}+\delta_{1}^{n+1}=0 \Rightarrow \delta_{-1}^{n+1}=-\left(4 \delta_{0}^{n+1}+\delta_{1}^{n+1}\right) \\
& U\left(x_{N}\right)=\delta_{N-1}^{n+1}+4 \delta_{N}^{n+1}+\delta_{N+1}^{n+1}=0 \Rightarrow \delta_{N+1}^{n+1}=-\left(\delta_{N-1}^{n+1}+4 \delta_{N}^{n+1}\right)
\end{aligned}
$$

By these substitutions, the equation (14) is turned out to be $N+1$ unknown at each level of the time $n$ in order to solve it using by Thomas algorithm.

\section{The Stability Analysis}

Now, the stability analysis is investigated by using very useful technique which is called Fourier stability method. Additionally the stability analysis is also used for various finite difference methods[22]. Considering the equation (13) and (14) together, then

$$
U_{m}^{n+1}-U_{m}^{n}-\alpha^{2} \Delta t(1-\theta)\left(U_{x x}\right)_{m}^{n}-\alpha^{2} \theta \Delta t\left(U_{x x}\right)_{m}^{n+1}=0
$$

is obtained. Now substituting (6) into (16) y ields

$$
\begin{aligned}
& \delta_{m-1}^{n+1}\left(1-\frac{6}{h^{2}} \alpha^{2} \theta \Delta t\right)+\delta_{m}^{n+1}\left(4+\frac{12}{h^{2}} \alpha^{2} \theta \Delta t\right)+\delta_{m+1}^{n+1}\left(1-\frac{6}{h^{2}} \alpha^{2} \theta \Delta t\right) \\
& =\delta_{m-1}^{n}\left[1+\frac{6}{h^{2}} \alpha^{2} \Delta t(1-\theta)\right]+\delta_{m}^{n}\left[4-\frac{12}{h^{2}} \alpha^{2} \Delta t(1-\theta)\right]+\delta_{m+1}^{n}\left[1+\frac{6}{h^{2}} \alpha^{2} \Delta t(1-\theta)\right]
\end{aligned}
$$

Using the Fourier stability method, then $\delta_{m}^{n}=\xi^{n} e^{i \alpha m}$ may be written where $i=\sqrt{-1}, h$ is the step size defined in Section 2 and $\alpha=\beta h$. By this equality, (17) can be reduced as

$$
\xi(A+\theta B)=A-(1-\theta) B
$$

where $A=2 \cos \mu+4, \quad B=\frac{12}{h^{2}} \alpha^{2} \Delta t(1-\cos \mu)$. Then the solution is stable for $\theta \in\left[\frac{1}{2}, 1\right]$ using the Equation (14), since the inequality $|\xi| \leq 1$ holds by the Fourier stability method.

Table 1. Comparison of the solutions for some $\alpha$ when $\Delta t=0.0001$ and $h=0.0125$

\begin{tabular}{cccccccc}
\hline$x$ & $t$ & $\begin{array}{c}\alpha=1 \\
\text { Numerical }\end{array}$ & $\begin{array}{c}\alpha=1 \\
\text { Analytical }\end{array}$ & $\begin{array}{c}\alpha=0.1 \\
\text { Numerical }\end{array}$ & $\begin{array}{c}\alpha=0.1 \\
\text { Analytical }\end{array}$ & $\begin{array}{c}\alpha=0.01 \\
\text { Numerical }\end{array}$ & $\begin{array}{c}\alpha=0.01 \\
\text { Analytical }\end{array}$ \\
\hline \multirow{6}{*}{0.25} & 0.4 & 0.01365 & 0.01364 & 0.67971 & 0.67973 & 0.70683 & 0.70683 \\
& 0.6 & 0.00190 & 0.00189 & 0.66645 & 0.66645 & 0.70669 & 0.70669 \\
& 0.8 & 0.00026 & 0.00026 & 0.67974 & 0.65342 & 0.70655 & 0.70655 \\
& 1.0 & 0.00004 & 0.00003 & 0.64065 & 0.64065 & 0.70641 & 0.70641 \\
& 3.0 & 0.00000 & 0.00000 & 0.52585 & 0.52589 & 0.70501 & 0.70501 \\
& & & & & & & \\
0.50 & 0.4 & 0.01931 & 0.01929 & 0.96126 & 0.96129 & 0.99961 & 0.99961 \\
& 0.6 & 0.00268 & 0.00268 & 0.94250 & 0.94250 & 0.99941 & 0.99941 \\
& 0.8 & 0.00037 & 0.00037 & 0.92408 & 0.92407 & 0.99921 & 0.99921 \\
& 1.0 & 0.00005 & 0.00005 & 0.90602 & 0.90601 & 0.99901 & 0.99901 \\
& 3.0 & 0.00000 & 0.00000 & 0.74309 & 0.74372 & 0.99704 & 0.99704 \\
& 0.4 & 0.01365 & 0.01364 & 0.67970 & 0.67973 & 0.70682 & 0.70683 \\
& 0.6 & 0.00190 & 0.00189 & 0.66645 & 0.66645 & 0.70669 & 0.70669 \\
& 0.8 & 0.00026 & 0.00026 & 0.65342 & 0.65342 & 0.70655 & 0.70655 \\
& 1.0 & 0.00004 & 0.00003 & 0.64065 & 0.64065 & 0.70641 & 0.70641 \\
\end{tabular}


Table 2. Comparison of the results for $t=0.1, \Delta t=0.00001, \alpha=1$

\begin{tabular}{ccccccc}
\hline$x$ & $h=0.1$ & $h=0.05$ & $h=0.025$ & $h=0.0125$ & $h=0.00625$ & Analytical \\
\hline 0.1 & 0.11425 & 0.11495 & 0.11513 & 0.11517 & 0.11518 & 0.11517 \\
0.2 & 0.21732 & 0.21865 & 0.21898 & 0.21907 & 0.21909 & 0.21907 \\
0.3 & 0.29911 & 0.30094 & 0.30140 & 0.30152 & 0.30155 & 0.30152 \\
0.4 & 0.35163 & 0.35378 & 0.35432 & 0.35446 & 0.35449 & 0.35446 \\
0.5 & 0.36972 & 0.37199 & 0.37256 & 0.37270 & 0.37273 & 0.37270 \\
0.6 & 0.35163 & 0.35378 & 0.35432 & 0.35446 & 0.35449 & 0.35446 \\
0.7 & 0.29911 & 0.30094 & 0.30140 & 0.30152 & 0.30155 & 0.30152 \\
0.8 & 0.21732 & 0.21865 & 0.21898 & 0.21907 & 0.21909 & 0.21907 \\
0.9 & 0.11425 & 0.11495 & 0.11513 & 0.11517 & 0.11518 & 0.11517 \\
\hline
\end{tabular}

\section{Numerical Results and Discussion}

In this section, we applied the method in Section 2 to problem (7) with (8) and (9) in order to solve it nu merically. Since than numerical results are obtained. In order to control the validity and efficiency of the numerical solutions of the equation (7) in the domain $[0,1]$ under the initial condition

(2) and the boundary conditions (3), we calculated the analytical solution of the equation (7) with the initial and boundary conditions. Here, the time step is taken as $\Delta t=0.0001$ in calculations. Mainly, Table 1 shows the both numerical solutions for some $\alpha$ and the analytical solutions to comparing with the data for some values of $t$. It is seen from table that the method with decreasing step size gives better approximate solutions than the others.

When fixed $t=0.1$ is taken in calculations with $\Delta t=0.00001$, the results are shown and compared for different values of $h$ in Table 2 . It is also seen from the table that the results calculated for $h=0.025$, $h=0.0125$ and $h=0.00625$ coincide with the exact solution, while those for $h=0.1, h=0.05$ are approached rapidly to the exact solutions.

It is observed by two tables that the approximate solutions obtained for different step sizes are closed to the exact solutions. To further corroborate the applicability of the presented method, numerical solution profile has been plotted in Figs. 1 for $h=0.1, \Delta t=0.01$.

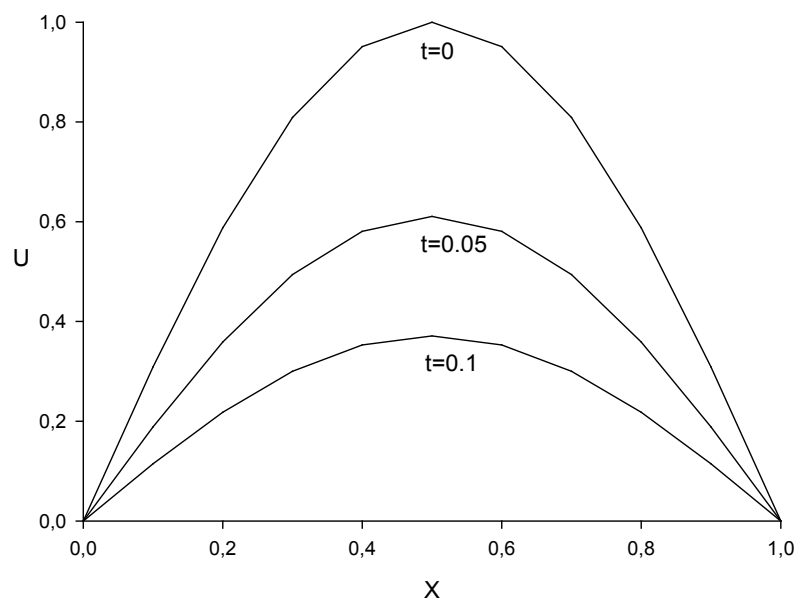

Figure 1. The numerical solution for $\alpha=1, h=0.1, \Delta t=0.01$

\section{Conclusions}

In this paper, we apply the collocation method with cubic B-splines finite elements to the heat equation success fully. In section 3, the stability of this method is analyzed considering Fourier stability method and it is found that the method is stable for $\theta \in[1 / 2,1]$. The results of the numerical solutions in Section 2 confirm that the accuracy, reliability and efficiency of the presented method which is applied in order to solve this type of the problem.

\section{REFERENCES}

[1] H.S. Carslaw , J.C. Jaeger, Conduction of Heat in Solids, Oxford University Pres., 1959.

[2] D.V. Widder, The Heat Equation, Academic Press, 1976.

[3] J.R. Cannon, The One-Dimensional Heat Equation, Cambridge University Pres., 1984.

[4] S. Dhawan, S. Kumar, A Numerical Solution of One Dimensional Heat Equation Using Cubic B-Spline Basis Functions, International Journal of Research and Reviews in Applied Sciences, 1 (1) (2009) 71-77.

[5] A.M. Wazwaz, Partial Differential Equations Methods and Applications, Saint Xavier University, 2002.

[6] en.wikipedia.org/wiki/Heat_equation.

[7] I. Dag, B. Saka, D. Irk, Application Cubic B-splines for Numerical Solution of the RLW Equation, Appl. Maths. and Comp., 159 (2004) 373-389.

[8] C. de Boor, "A Practicle guide to splines", Applied Mathematical Sciences, Springer-Verlag, 2001.

[9] C. de Boor, "On Calculating with B-splines", Journal of Approximation Theory, 6 (1972) 50- 62.

[10] S. Kutluay , A.R. Bahadır, A. Özdeş, Numerical Solution of One-dimensional Burger Equation: Explicit and Exact-explicit Finite Difference Methods, J. Comp. App. Math., 103 (1999) 251-261.

[11] I. Dag, D. Irk, B. Saka, A Numerical Solution of the Burgers' Equation Using Cubic B-splines, Appl. Maths. and Comp., 163 (2005) 199-211.

[12] S.G. Rubin, P.K. Khosla, Higher-Order Numerical Solutions Using Cubic Splines, AIAA J., 14 (1976) 851-858. 
[13] H. Caglar, M. Özer, N. Caglar, The Numerical Solution of the One-dimensional Heat Equation by Using Third Degree B-spline Functions, Chaos, Solitons \& Fractals, 38 (2008) $1197-1201$.

[14] A.R. Bahadır, Application of Cubic B-spline Finite Element Technique to the Thermistor Problem, Applied Mathematics and Computation, 149 (2004) 379-387.

[15] B. Saka, I. Dag, Quartic B-spline Collocation Method to the Numerical Solutions of the Burgers' Equation, Chaos, Solitons \& Fractals, 32 (2007) 1125-1137.

[16] B. Saka, I. Dag, A. Boz, B-spline Galerkin Methods for Numerical Solutions of the Burgers' Equation, Appl. Maths. and Comp., 166 (2005) 506-522.

[17] M.A. Ramadan , T.S. El-Danaf , F.E.I. Abd Alaal, A Numerical Solution of the Burgers' Equation Using Septic B-splines, Chaos, Solitons \& Fractals, 26 (2005) 795-804.
[18] S.G. Rubin, R.A. Graves, Cubic Spline Approximation for Problems in Fluid Mechanics, Nasa TR R-436, Washington, DC, 1975.

[19] P.M. Prenter, Splines and Variational Methods, John Wiley, New York, 1975.

[20] A.K. Khalifa, K.R. Raslana, H.M. Alzubaidi, A Collocation Method with Cubic B-splines for Solving the MRLW Equation, Journal of Computational and Applied Mathematics 212 (2008) $406-418$.

[21] A.H.A. Ali , L.R.T. Gardner, G.A.Gardner, A Collocation Method for Burgers' Equation Using Cubic Splines, Comp. Math. Appl. Mech. Eng., 100 (1992) 325-337.

[22] G. Sewell, The Numerical Solution of Ordinary and Partial Differential Equations, John Wiley and Sons, 2005. 